\title{
Corela
}

Cognition, représentation, langage

HS-19 | 2016

Le point de vue pris au mot

\section{Sémantique instructionnelle : propositions et études de cas}

\section{Gilles Col et Jeanne Aptekman}

\section{(2) OpenEdition}

1 Journals

\section{Édition électronique}

URL : https://journals.openedition.org/corela/4407

DOI : $10.4000 /$ corela.4407

ISSN : 1638-573X

Éditeur

Cercle linguistique du Centre et de l'Ouest - CerLICO

Référence électronique

Gilles Col et Jeanne Aptekman, «Sémantique instructionnelle : propositions et études de cas », Corela [En ligne], HS-19 | 2016, mis en ligne le 12 octobre 2016, consulté le 18 mars 2022. URL : http:// journals.openedition.org/corela/4407 ; DOI : https://doi.org/10.4000/corela.4407

Ce document a été généré automatiquement le 18 mars 2022.

\section{(c) (i) (2)(2)}

Corela - cognition, représentation, langage est mis à disposition selon les termes de la licence Creative Commons Attribution - Pas d'Utilisation Commerciale - Partage dans les Mêmes Conditions 4.0 International. 


\title{
Sémantique instructionnelle : propositions et études de cas
}

\author{
Gilles Col et Jeanne Aptekman
}

1 Notre contribution rend hommage à une partie des travaux de Pierre-Yves Raccah, en l'occurrence aux études qu'il a consacrées à la sémantique des points de vue. L'idée générale de la théorie de Raccah est que la signification des mots d'une langue donnée comprend des contraintes sur la manière dont l'interlocuteur doit percevoir ces mots. Ces contraintes s'apparentent à des instructions sémantiques ou tout du moins à un des aspects que prennent ces instructions dans le traitement des unités linguistiques et dans leurs interprétations. Dans ses travaux, Raccah a développé de manière approfondie le cas des unités qu'il appelle "articulateurs » comme " mais », «trop », «assez »; ces unités relèvent très clairement de ces instructions et c'est donc dans cette lignée de travaux que notre contribution s'inscrit. Comme le fait Raccah (2005) pour qui «il est donc nécessaire d'admettre que d'autres mots que les articulateurs imposent des contraintes sur les points de vue et, cette fois, non pas sur les articulations, mais sur la nature des points de vue.» (Raccah 2005: 178), nous proposons d'étendre la notion d'instruction au-delà des seuls articulateurs et dans ce but, nous consacrons les lignes qui suivent aux unités grammaticales et à leurs interactions.

\section{Instructions dynamiques de construction du sens : hypothèses}

2 Notre point de départ consiste ici à considérer que le sens d'une expression dans un énoncé donné est composé d'une part de son apport propre et d'autre part de ses règles d'interaction avec le reste de l'énoncé. Cette thèse n'a rien d'original, et a été développée par de nombreux auteurs, notamment Benveniste ${ }^{1}$ ou bien encore, dans un champ très différent, en DRT $^{2}$ et dans les courants proches de la linguistique dite cognitive. Elle s'appuie sur l'idée selon laquelle « une partie dans un tout est tout autre chose que cette partie isolée ou dans un autre tout » (Guillaume [1919] 1979 : 23). 
3 Mais en considérant la construction du sens comme un système dynamique, nous faisons l'hypothèse que l'on peut décrire cette construction dans sa progression même, au fur et à mesure de la perception des unités linguistiques et de leur traitement cognitif. Le contexte prend alors un rôle fondamental: la progression sémantique, si elle est dynamique, implique effectivement la déformation d'unités du contexte perçues et traitées dans un premier temps. Cela a comme conséquence que ce qu'apporte chaque unité à la construction du sens d'un énoncé doit être défini selon un principe qui permet de prévoir ce qu'apporte l'unité à la construction et ce qu'elle reçoit de la construction.

4 Notre but est donc de montrer, à partir d'exemples tirés de corpus ${ }^{3}$, comment cette double action (de l'unité sur la l'énoncé, et inversement, de l'énoncé sur l'unité) peut être analysée. Évidemment, ce travail est voué à sortir des frontières de l'énoncé pour prendre en compte le contexte de manière beaucoup plus large, mais c'est au contexte intra-énoncé et aux relations dynamiques des unités entre elles à l'intérieur d'un énoncé que nous allons nous intéresser ici. Les «bords" de l'énoncé seront pris en compte également, mais dans leurs relations avec certains éléments provenant d'autres scènes verbales, notamment à travers la question du rôle des pronoms.

5 Nous analyserons ainsi la progression dynamique de la construction sémantique de quelques exemples, en français et en anglais. Cette analyse passe par la prise en compte de l'instruction fournie par chaque unité de l'énoncé, instruction que nous appelons ici "forme schématique» et que nous définissons sur le principe de convocation / évocation (Victorri 1996, 1999).

6 Avant de revenir sur les problèmes méthodologiques posés par une telle approche, nous voudrions définir les notions d'« instruction » et de «forme schématique » sur lesquelles nous nous appuyons.

\subsection{Instructions et espaces mentaux}

7 Notre hypothèse consiste à penser les unités linguistiques comme fournissant une instruction pour l'interprétation de l'énoncé en contexte. La notion d'« instruction » est en fait assez largement partagée en sémantique cognitive, même si elle peut prendre diverses formes. Pour Raccah (2011) par exemple, cette notion est appliquée aux mots et aux syntagmes et elle en constitue leur signification. Elles ont également un rôle que l'on pourrait qualifier d'«indicatif» dans la construction du sens d'un énoncé :

Les mots de la langue, que le locuteur choisit d'utiliser pour son énoncé, indiquent à l'interlocuteur comment il est censé utiliser les éléments de sa conception de la situation pour construire le sens de l'énoncé. Ces indications, qui se propagent des mots aux syntagmes et aux phrases, et contraignent la construction du sens des énoncés, peuvent être conçues comme des instructions: elles constituent la valeur sémantique des unités de langue : leur signification. (Raccah 2011)

Chez Raccah, les instructions ont ainsi la double caractéristique d'être des indications pour la construction du sens et des contraintes car elles infléchissent d'une certaine façon la construction du sens. Cette notion s'applique par ailleurs à toutes les unités d'un énoncé, ce que certaines approches sémantiques cognitives ne partagent pas toujours. C'est le cas par exemple chez Fauconnier et sa théorie des Espaces Mentaux. Fauconnier s'intéresse effectivement à ce qu'il appelle des " constructeurs d'espaces ", unités qui donnent des indications et des indices sur le déploiement des réseaux 
d'espaces mentaux. Fauconnier limite cependant ce rôle de " constructeur » à certaines expressions linguistiques, essentiellement des expressions grammaticales comme les temps et aspects, les syntagmes prépositionnels adverbiaux ( «In 1929,...»), les constructions verbales à rôle modal («Susan believes...», "Max hopes...»), les subordonnées conjonctives ( If it trains... »). Dès lors, cette notion de constructeur d'espaces se retrouve sous-tendue par la distinction posée entre sens grammatical et sens lexical ${ }^{4}$. Or, contrairement à l'interprétation qui est parfois faite de cette distinction, nous voudrions comprendre celle-ci non comme opposant deux types d'unités, codant deux types d'instructions différents, mais comme permettant de penser que toutes les unités participent à la construction dynamique du sens. En conséquence, une même unité, notamment en ce qui concerne les verbes, peut avoir à la fois un sens grammatical et un sens lexical.

9 Dans cette perspective, l'instruction fournie par une unité est unique, mais elle peut avoir des effets très variés suivant le type de configuration cognitive (ou de réseau d'espaces de connaissances) sur laquelle elle opère. Là encore, nous rejoignons le point de vue de Fauconnier (1997) :

The space-building instructions associated with a particular grammatical construction are unique. [...] But the effects of such instructions may be widely different, depending on the configuration they operate on when they come into the discourse. (Fauconnier 1997 : 65) [souligné dans le texte]

10 Ainsi, même si nous ne reprenons pas à notre compte toute l'analyse de Fauconnier, dont nous nous éloignons seulement en tant que nous privilégions un continuum entre sens lexical et grammatical, nous voudrions garder l'idée centrale de leur caractère sous-spécifié :

The language form contains underspecified instruction for space building. It can apply to infinitely many kinds of input; and for any given input there is a finite number of outputs that it can yield. (idem : 65) [souligné dans le texte]

11 Cette notion de sous-spécification est en fait centrale dans l'analyse de Fauconnier, du fait qu'il considère un espace mental comme une structure partielle prise dans un réseau d'espaces qui s'enrichit dans le déroulement du discours et permet le déploiement du sens. Ainsi,

[...] une expression de langue qui intervient dans le discours au stade $\underline{n}$ place alors un ensemble de contraintes sur la nouvelle configuration produite, cela en fonction de la configuration déjà engendrée au stade n-1. (Fauconnier 1991 : 231)

Le développement des espaces, leurs relations et leur changement de statut au fur et à mesure que le discours se déroule ("Base ", «Focus ", "Point de vue » pour les plus importants, cf. Fauconnier 1997, ou Col 2010b) leur donnent un caractère dynamique et contribuent à faire de la construction du sens par instruction un processus lui-même dynamique. La notion d'instruction que nous adopterons dans cette étude se distingue de celle développée par Fauconnier. Le terme même d'«instruire » sera effectivement utilisé minimalement au sens de fournir des connaissances, des informations ou des directives. Dans le domaine de l'informatique par exemple, une instruction est une expression qui spécifie une opération. La définition qu'on peut en lire dans un dictionnaire comme le Oxford English Dictionary est la suivante :

INSTRUCTION: Computers. An expression in a program or routine, or a sequence of characters in a machine language, which specifies an operation (esp. a basic operation) and freq. also one or more operands, and results in its performance by the computer. (Oxford English Dictionary) 
Une instruction correspond par conséquent à la «consigne » donnée par une unité linguistique à la construction globale du sens de l'énoncé. Cette consigne, comme on le voit plus bas, s'exprime en termes de convocation / évocation; elle correspond par ailleurs à la « forme schématique » de l'unité.

\subsection{Instruction et forme schématique}

14 Comme nous l'avons mentionné, si nous souhaitons reprendre à notre compte cette notion d'instruction, il s'agit cependant pour nous de considérer que toutes les unités linguistiques fournissent des instructions récusant ainsi une distinction a priori entre unités grammaticales et unités lexicales, qui verrait les seules unités grammaticales comme instructionnelles ${ }^{5}$.

L'instruction associée à une unité linguistique peut se comprendre comme étant fournie par la «forme schématique» (Culioli 1990) des unités, c'est-à-dire une représentation métalinguistique associée à une forme empirique. Cette notion de " forme schématique ", telle que développée par Culioli (1990) permet de tenir compte des variations sémantiques régulées des unités linguistiques, et propose un principe dynamique de déformation. Elle repose sur le constat suivant :

Les phénomènes linguistiques forment des systèmes dynamiques qui sont réguliers, mais avec une marge de variation due à des facteurs d'une grande diversité : on a affaire à des phénomènes qui sont à la fois stables et plastiques. [...] La déformation est une transformation qui modifie une configuration, de sorte que certaines propriétés restent invariantes sous transformation, tandis que d'autres vont varier. [...] Pour qu'il y ait déformabilité, il faut que l'on ait affaire à une forme schématique (telle qu'il puisse y avoir à la fois modification et invariance), que l'on ait des facteurs de déformation et que l'on ait une marge de jeu, un espace d'ajustement muni de propriétés topologiques. (Culioli 1990 : 129-130) [souligné dans le texte]

En reprenant cette définition, ainsi que la notion de sous-spécification des instructions développée par Fauconnier, nous considérons que la forme schématique des unités linguistiques doit être formulée de façon à pouvoir prévoir son assemblage avec les autres formes schématiques des autres unités présentes dans l'énoncé. Si effectivement, le "sens » répond à une construction, chaque unité linguistique y joue un rôle et a ainsi besoin d'autres unités pour pouvoir jouer pleinement son rôle d'évocation d'« incréments sémantiques ".

Notre objectif étant de décrire, pour un énoncé donné, la construction du sens dans son déroulement même, nous postulons non seulement que la construction du sens peut se faire par la description de "formes schématiques ", comme le suggèrent les travaux de Culioli, mais également que ces formes schématiques s'assemblent pour construire du sens de l'énoncé. Mais ces « incréments sémantiques » ne s'assemblent pas au hasard, et nous voudrions montrer comment les formes schématiques elles-mêmes fournissent des instructions pour cet assemblage dynamique.

En effet, une forme schématique doit prévoir, dans sa formulation, son assemblage avec une autre forme, reflétant ainsi la mise en place des relations prédicatives d'un énoncé. Fondamentalement une forme schématique doit contenir la capacité de convoquer certains éléments pour que l'unité puisse vraiment jouer son rôle. 


\subsection{Définitions}

19 Avant de décrire étape après étape la construction du sens d'un énoncé en anglais et d'un énoncé en français, nous voudrions apporter certaines précisions méthodologiques et définitionnelles.

\subsubsection{Convocation et évocation}

Dans la lignée de nos travaux précédents ${ }^{6}$, nous nous appuyons, pour déterminer la forme schématique des éléments, sur des opérations de convocation et d'évocation. Le principe de convocation-évocation est défini (Victorri 1999 ; Col 2008b) comme régissant la façon dont chaque unité interagit avec le contexte et le co-texte pour jouer son rôle de construction. Il permet de rendre compte d'un double mouvement qui correspond aux deux étapes par lesquelles on peut décrire le sens d'une unité, c'est-à-dire :

d'une part déterminer ce qui doit être présent dans le champ intersubjectif (y compris la scène en train de se construire) pour que l'unité puisse jouer son rôle dans cette construction: ces éléments, nécessaires au bon fonctionnement de l'unité, mais dont elle n'est pas elle-même porteuse, nous dirons qu'elle les convoque, et d'autre part déterminer ce que l'unité apporte à la construction en agissant sur les éléments qu'elle a convoqués; cette action a un effet sur la scène verbale en construction : c'est cela que l'unité évoque. (Victorri 1999 : 96)

Dans la perspective présentée ici, une forme schématique met en œuvre les deux aspects du principe de convocation-évocation, y compris dans sa formulation (voir 2.1 pour l'exemple de la forme schématique instructionnelle de «always »).

\subsubsection{Représentation, entités, procès}

terme de « représentation » va prendre ici le sens de mise en présence sur le champ intersubjectif, c'est-à-dire la scène en construction, d'entités et de procès ${ }^{7}$. Le locuteur choisit de présenter sur la scène verbale des événements, des raisonnements, des émotions, des notions, etc., sous la forme soit d'entités, soit de procès.

ar définition, une entité est topologiquement individuable et stable dans son évolution sur la scène, alors qu'un procès présente les relations entre les entités et ces relations évoluent dans la temporalité de la scène ${ }^{8}$.

es entités et les procès sont en fait ce qu'évoquent les unités linguistiques convoquées sur la scène en construction. On considère que ces notions, sentiments, etc., sont "étiquetés » par les unités linguistiques.

\subsubsection{Systématicité des formes schématiques}

Enfin, il est nécessaire de définir ces formes schématiques de manière systématique, comme une grammaire contenant un ensemble de règles, et sur un principe identique pour chaque unité afin d'en permettre l'assemblage. On pourra alors parler de "grammaire instructionnelle» sur le principe de convocation / évocation. Cela est d'autant plus essentiel dans notre approche qui revendique une participation de tous les éléments à la construction dynamique du sens. 


\subsection{La question de l'ordre de traitement des unités}

l'information ». La notion d'« information » est à prendre dans un sens général ; elle recouvre tous les éléments écrits ou oraux qui participent à l'élaboration des représentations et qui font intervenir les connaissances du sujet. C'est en fait le principe cognitif fondamental à l'œuvre dans ce traitement qui est central ici. Ce principe cognitif est que les informations fournies par les unités linguistiques (lexicales, grammaticales, prosodiques) sont traitées au fur et à mesure du déroulement de l'énoncé ; la scène verbale se construit ainsi «à la volée » au fur et à mesure de la production de l'énoncé. Chaque unité joue son rôle d'évocation dès que les éléments qu'elle convoque sont disponibles. Le processus est cependant loin d'être linéaire. Si les éléments que convoque une unité ne sont pas (encore) présents sur la scène en construction, et donc s'ils sont non disponibles, l'unité ne peut pas jouer son rôle d'évocation. D'où cette idée de « mise en attente » jusqu'à ce que les éléments dont elle a besoin pour contribuer à la construction du sens de l'énoncé soient présents sur la scène. On est donc bien dans une perspective dynamique et non linéaire. D’ailleurs, une fois leur travail d'évocation effectué, les éléments de la scène en construction continuent d'être déterminés, voire transformés lors de convocations ultérieures par d'autres unités ; ainsi le sens des unités polysémiques se précise - et se "profile » pour reprendre une expression de la grammaire cognitive (Langacker 1987) progressivement.

31 Les informations ainsi traitées sont ensuite assemblées dans une représentation générale comme on l'a vu dans la première partie, ou plutôt dans des représentations qui vont permettre la compréhension de l'énoncé. La question de l'ordre dans lequel ces informations sont traitées est en fait le cœur du problème. De manière générale, 
l'ordre de traitement n'est pas fixé une fois pour toutes, mais afin de définir un cadre d'analyse, nous avons été amenés à adopter les trois grands principes suivants (Col 2008b) :

- ${ }^{\text {er }}$ principe de traitement: les unités sont prises en compte au fur et à mesure qu'elles apparaissent dans la perception de l'énoncé.

- $2^{\text {ème }}$ principe : une fois prises en compte, si elles peuvent être traitées, elles le sont tout de suite conformément à leur forme schématique. Ainsi, chaque unité joue son rôle d'évocation dès que les éléments qu'elle convoque sont disponibles.

- $3^{\text {ème }}$ principe : si elles ne peuvent pas être traitées, elles sont mises en attente jusqu'à ce que les éléments qu'elles convoquent soient introduits sur la scène en construction.

À ces trois principes, on peut en ajouter un autre qui concerne indirectement l'ordre de traitement en lui conférant son caractère dynamique et non linéaire :

- $4^{\text {éme }}$ principe : une fois évoqués, les éléments de la scène en construction continuent d'être déterminés, voire transformés lors de convocations ultérieures par d'autres unités.

Dans cette optique, les mots ont un rôle structurant: ils permettent aux notions (ensemble de propriétés physico-culturelles d'ordre cognitif et générateur d'unités lexicales) ${ }^{9}$ d'être structurées et lexicalisées.

Le point central de notre analyse est que tous les mots, indépendamment de leur sens lexical ou grammatical, ont un rôle relationnel: ils permettent la mise en relation d'entités et de procès.

Si on prend comme exemple le groupe nominal « un goûter ", la première unité qui se présente à la perception est l'article indéfini «un ». L'instruction fournie par cette unité est de convoquer une "notion d'entité », c'est-à-dire une notion indéterminée mais susceptible d'être profilée en entité, que l'on note e, et d'évoquer une véritable entité issue de cette notion par extraction et notée $\mathrm{E}_{\mathrm{n}}{ }^{10}$. Mais au moment du traitement de « un ", il n'y a pas d'autres éléments sur la scène, donc cette unité ne peut pas être traitée complètement et va être mise en attente; elle ne peut rien convoquer. C'est seulement au moment où "goûter » est pris en compte dans la perception et donc au moment où il est introduit sur la scène sous forme de notion indéterminée que " un " va exercer son rôle convocateur. Le traitement de "goûter », sous l'effet de l'unité « un », correspond alors à sa " transformation » ou " profilage » en entité nominale. En effet, l'instruction fournie par « un » est de convoquer une «notion d'entité » comme on a dit plus haut, et d'évoquer une véritable entité issue de cette notion par extraction et notée $E_{n}$. On considère effectivement qu'un mot grammatical est porteur d'une opération métalinguistique, ici l'opération complexe d'« extraction » qui correspond en fait à une série d'opérations passant par le repérage d'une notion par rapport à une situation d'énonciation, le prélèvement d'un objet effectué sur cette notion et enfin la détermination quantitative de cet objet (Culioli 1999: 46-47). L'instruction donnée par « un » est fondamentalement d'évoquer une entité et son passage de la notion /goûter/ (pour reprendre la notation proposée par Culioli), que l'on note $\mathrm{n}_{\mathrm{p} / \mathrm{n}}$ à l'entité $\mathrm{E}_{\mathrm{n}}$.

\section{Illustrations et descriptions d'exemples}

Pour illustrer cette approche, nous allons ici présenter l'analyse de deux exemples différents, en anglais ${ }^{11}$ et en français. 


\subsection{Exemple en anglais}

\section{explicitement ou implicitement comme en (2):}

(2) My task has been to reconsider some of those complicated but always significant histories wherein differences conflict and converge as desire itself. (British National Corpus A6D 1841)

[Ma tâche est de réanalyser certaines histoires compliquées mais toujours importantes, dans lesquelles les différences convergent et divergent, comme le désir lui-même.]

Il peut également avoir un sens intensifieur (Quirk et alii 1985 : 588-589), notamment quand il apparaît avec un modal comme can :

(3) But I'm very young, and if it goes wrong, I can always do something else. (BNC ABJ 1790)

[Mais je suis très jeune et si les choses tournent mal, je peux toujours faire autre chose.]

Dans ce type d'exemple, always prend alors un sens non pas temporel, mais plutôt «modal »: il indique ce qu'on pourrait appeler un «renforcement» de la bifurcation construite par le modal en introduisant l'idée de suggestion. Pour Gilbert (2001), ce type d'exemple correspond au cas où « le parcours de la classe des instants marqué par l'adverbe always reflète explicitement la présence sous-jacente d'une classe d'occurrences" (Gilbert 2001: 49). Parler de "parcours de la classe des instants " correspond à des co-textes comme celui de (3), mais correspond aussi à l'exemple suivant :

(4) God is infinitely potential: his is a dynamic power, from which an infinite set of new and different values can always arise. (BNC AMT 1431)

[Dieu est infiniment tout puissant : c'est un pouvoir dynamique à partir duquel un ensemble infini de valeurs nouvelles et différentes peuvent toujours émerger.]

Bien que contenant le modal "can» comme en (3), ce dernier exemple évoque une propriété "sporadique» du sujet, dans le sens où cette propriété (c'est-à-dire, l'émergence de nouvelles valeurs) se manifeste de temps en temps et dans certaines conditions. Le point commun à ces trois exemples n'en reste pas moins que les 
éléments considérés (des instants en (2) et (4), des propriétés en (3)) sont regroupés dans une classe où ils sont en quelque sorte "mis à égalité ». On peut effectivement parler de "parcours» de cette classe, pour reprendre l'opération développée dans la Théorie des Opérations Prédicatives et Énonciatives; dans cette perspective, la caractéristique de always est alors d'évoquer que ce parcours est continu. Si on le compare à l'adverbe still, qui évoque une trajectoire et une orientation sur cette trajectoire - avec l'idée qu'il y a encore un procès, une propriété, ou un moment attendus -, on constate qu'avec always il n'y a pas de moment ou de procès attendus. Le rôle de cet adverbe est de convoquer un domaine cognitif, une prédication sur ce domaine, et un parcours sur le domaine. Il évoque alors qu'il y a continuité sur le domaine, ou plus exactement qu'il y a continuité du prédicat sur le parcours du domaine cognitif. Une ébauche de forme schématique pour always ${ }^{12}$ pourrait être formulée de la façon suivante :

- Always : convoque un domaine, une prédication sur ce domaine et un parcours sur le domaine ; évoque qu'il y a continuité du prédicat sur le parcours.

À partir de cette forme schématique, la plus problématique, nous pouvons décomposer l'analyse de cet exemple en plusieurs «moments », qui correspondent aux différentes étapes de traitement que nous pouvons identifier - mais qui ne seront pas toujours totalement distinguables les uns des autres compte tenu de la quasi-simultanéité de la perception des unités. La notion de "moment " est en apparence simplificatrice et pourrait donner l'illusion d'une forme de compositionnalité. Rappelons malgré tout que notre objectif est de décrire et de formaliser la construction du sens dans son déroulement et dans sa temporalité propre. La notion de "moment » peut alors être perçue comme un artefact pour l'analyse et n'empêche aucune simultanéité ni "retour", comme on va pouvoir le constater (notamment en ce qui concerne le traitement des unités verbales).

Reprenons l'exemple (1):

(1) You've always been controversial.

On peut le décomposer en différentes unités de la façon suivante :

YOU HAVE ALWAYS BE -EN ${ }^{13}$ CONTROVERSIAL

Nous faisons l'hypothèse de six moments différents représentant six étapes de traitement différentes pour cet énoncé :

Moment 1 : La première unité qui se présente à la perception est le pronom personnel sujet You. Un pronom comme celui-ci convoque par définition sur la scène une entité humaine E la plus saillante et identifiée au co-énonciateur. Celui à qui le pronom fait référence est directement situé sur la scène en construction. Il est construit et évoqué immédiatement comme entité sur la scène, d'où sa notation en $\mathrm{E}$ (voir note 9). Donc You, de part sa forme schématique, fait venir sur la scène une première entité $E_{1}$ correspondant au co-énonciateur et issue par exemple de la notion /Pedro/, c'est-àdire la personne à laquelle renvoie le pronom, et elle évoque cette entité.

Cette entité va être convoquée ensuite deux fois. Mais à ce stade, le travail d'évocation des unités qui suivent ne peut pas se faire: HAVE et BE ont chacune besoin de deux éléments (entité ou procès) pour effectuer leur travail d'évocation - ces unités correspondent à des auxiliaires -, or une seule entité est présente sur la scène. Elles sont donc mises en attente. 
51 Moment 2: L'unité conTROVERSIAL peut être traitée avant les autres : cette unité rend présente sur la scène une notion et évoque une propriété issue de cette notion, $\mathrm{q}_{1}$. La présence de cette propriété sur la scène permet alors à l'unité BE d'effectuer son travail de convocation et d'évocation.

52 Moment 3: $\mathrm{BE}$ accroche alors la propriété $\mathrm{q}_{1}$ et l'entité $\mathrm{E}_{1}$ et évoque que ces deux éléments sont identifiables. L'identification ne peut cependant pas se faire sans être située temporellement; pour l'instant, il y a identification possible. Il y a surtout que cette identification est évoquée comme un état résultant par -EN qui agit sur la scène quasiment simultanément.

53 Moment 4-5: -EN va chercher l'identification et évoque que celle-ci est un état résultant, ou plus précisément, que c'est une propriété résultante d'une notion de procès. À un second niveau, et quasiment simultanément, HAVE fait appel à $\mathrm{E}_{1}$, ainsi qu'à ce qui a été évoqué juste avant. Et c'est l'ensemble, tout le "complexe verbal ", qui évoque un procès correspondant à l'identification localisée (située par rapport à un moment différent de $\mathrm{T}_{0}$ ) d'une propriété issue de la notion /debate/ avec l'entité $\mathrm{E}_{1}$ provenant d'une scène différente. Le travail de ALWAYs peut alors se faire.

54 Moment 6 : ALWAYS a besoin, conformément à sa forme schématique, d'un domaine et d'une prédication; il convoque aussi un parcours sur ce domaine. Le domaine est présent sur la scène (/debate/, profilé sous la forme de la propriété évoquée par « controversial »), la prédication aussi (You = sujet de polémique).

ALWAYS fait par ailleurs appel à un intervalle situé temporellement et évoqué par « have been controversial». L'intervalle est construit par « have been controversial» et représente une classe d'occurrences de «be controversial ». Les différents " états » ou différentes identifications de you et controversial construisent une classe d'occurrences par opposition à un énoncé comme «You are controversial» ou «You were controversial» qui n'évoquent que des états - des procès inactivés - situés hors situation d'énonciation.

56 ALWAYs évoque alors la continuité sur le parcours, c'est-à-dire, (1) la validation pour chaque moment de l'intervalle de l'identification you / controversial, et (2) la poursuite de la prédication en dehors de l'intervalle (d'où la notation où les barres sont en pointillés).

57 Nous pouvons à présent tenter de synthétiser la construction progressive de la représentation sémantique de l'énoncé (1) de la façon suivante : 


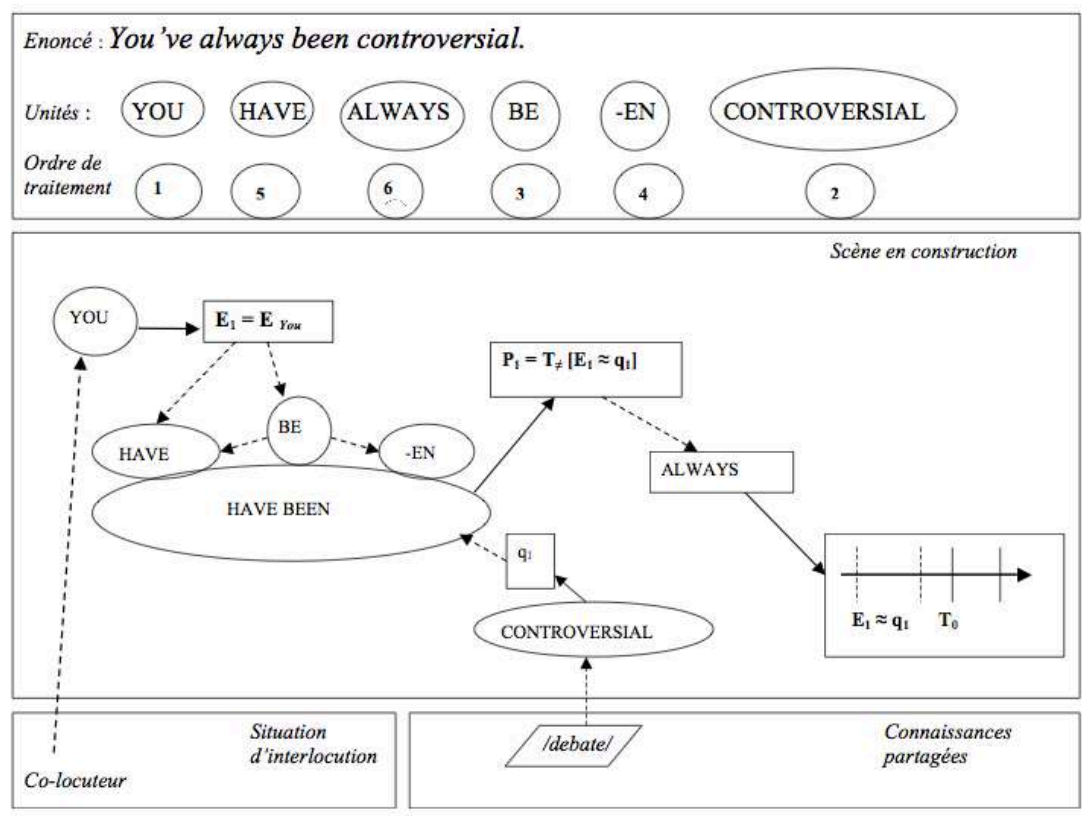

Fig. $n^{\circ} 1$ : progression sémantique de l'énoncé : « You've always been controversial »

Les flèches en pointillés signalent ce qu'une unité convoque sur la scène, tandis que les flèches pleines signalent ce qu'elles évoquent. Par ailleurs, les chiffres situés sous chacune des unités correspondent à l'ordre de traitement de ces unités. Ils ont un caractère purement informationnel dans la mesure où on a constaté plus haut que deux étapes de la construction du sens pouvaient parfois être simultanées (comme la 5 et la 6). On remarque également que l'ordre de traitement ne correspond pas à l'ordre des unités, excepté la première unité qui, en tant que pronom personnel, est une unité qui fait le lien avec la situation d'énonciation et permet d'ancrer l'énoncé sur la scène verbale.

La question de l'ordre de traitement des unités reflète en fait une question plus profonde qui est celle du type de calcul du sens à l'œuvre dans notre modèle. Ce type de calcul n'est pas celui de la compositionnalité classique qui s'effectue dans un ordre donné et qui permet de calculer de proche en proche le sens global de l'énoncé à partir des éléments le constituant. Le type de calcul adopté ici est plutôt celui d'une compositionnalité plus générale, ou compositionnalité " gestaltiste » qui repose sur un mécanisme cognitif commun à la perception visuelle. Victorri (1996:174-182) relève en effet une analogie entre l'interprétation d'un dessin et celle d'un énoncé et reconnaît que certains mécanismes à l'œuvre dans l'activité de langage pourraient être communs à d'autres activités cognitives. Ce constat lui permet alors de proposer un calcul de type compositionnel mais reposant sur un mécanisme cognitif présent dans la perception visuelle :

[...] les interactions entre éléments [d'un énoncé] ne sont pas a priori décomposables, et le calcul doit donc avoir un caractère global en prenant simultanément en compte les influences réciproques (Victorri 1996:178) [...] le sens que l'on attribue à tel ou tel élément dans un énoncé est composé de la combinaison de son apport propre et de l'apport du reste de l'énoncé : c'est donc bien une propriété globale de l'énoncé. (Victorri 1996 : 181) 
Ainsi, le sens aspectuel perfectif évoqué dans l'exemple (1) traité précédemment est une caractéristique globale qui ne repose pas sur un seul élément mais sur plusieurs, comme le montre la figure synthétique ci-dessus. De la même façon, il n'existe pas dans ce modèle un mécanisme de hiérarchisation correspondant aux regroupements de syntagmes. L'exemple (1) montre que l'on peut retrouver de tels regroupements, en l'occurrence au niveau du syntagme verbal. Ce regroupement ne correspond cependant pas à un calcul hiérarchisé mais plutôt à un couplage d'éléments qui s'impose à la perception d'autant que d'autres éléments (ALWAYS, CONTROVERSIAL) sont en attente et ne peuvent pas être traités avant ce complexe verbal.

61 Ainsi l'analyse d'un tel exemple permet déjà de se faire une idée des difficultés rencontrées si l'on veut rendre compte de la construction dynamique du sens. Ces difficultés concernent plusieurs domaines :

- la question de l'ordre de traitement et celle de son caractère « compositionnel gestaltiste ». C'est là que la problématique du contexte est la plus pertinente ;

- la définition des instructions fournies par les unités, et donc des formes schématiques à l'origine de ces instructions. Ces instructions sont dynamiques et doivent créer des relations fortes entre les unités d'un énoncé, d'où leur degré de généralité élevé, l'accent étant mis sur leur caractère dynamico-relationnel avant tout ;

- la question de l'origine des éléments convoqués sur la scène. La notion de « connaissances partagées » par exemple est très générale et demande un cadre définitoire plus élaboré.

\subsection{Exemple en français}

(5) Voilà la photo que j'ai préférée.

La particularité de cet exemple est la présence de la subordonnée relative, mais avant d'aborder ce point, une autre question se pose : celle de l'instruction fournie par l'unité voILÀ. Il se trouve que cette unité est de manière assez évidente plurifonctionnelle et polysémique. Les travaux menés jusque là font d'elle une unité relativement inclassable, de l'ordre du "gallicisme» (Léard 1992). Assimilé à la notion de "présentatif », le terme est autant considéré comme un adverbe que comme un verbe invariable défectif. Il joue aussi un rôle qui le rapproche d'une préposition, marquant une relation temporelle d'antériorité («ils ont commencé leur ascension voilà huit jours »), et il peut être employé comme une interjection (« Voilà !»). L'ambiguïté de son statut et les insuffisances de la catégorisation traditionnelle sont particulièrement sensibles dans les tentatives de dénominations, plus ou moins hybrides : mi adverbe, mi préposition (Le Bidois 1956; Grévisse 1980), adverbe présentatif (Brunot et Bruneau 1969), « une sorte de verbe » et « une sorte de préposition » (Moignet 1969), une particule démonstrative (Wagner et Pinchon 1962), a subjectless verb (Morin 1985).

La valeur monstrative de voilà, qui s'explique morphologiquement par l'impératif ( "vois») et le déictique («là»), en fait aussi une unité autonome, non-prédicative, «marqueur de structuration de conversation» (Auchlin 1981) ce qui la rapproche des «mots du discours » (Hansen 1997). De manière synthétique, on peut distinguer deux axes (l'axe présentatif et l'axe déictique (Grenoble et Riley 1996) et plusieurs voilà: le voilà prédicatif, le voilà prépositionnel et interjectif (Narjoux 2003), le «voilà présentatif », le voilà "polyphonique » (Delahaie 2013). Cette distinction peut 
également être tripolaire : voilà est soit une préposition, soit un présentatif, soit un marqueur de discours (Porhiel 2012).

Sur le plan sémantique qui nous concerne plus particulièrement, voilà possède indéniablement une valeur monstrative, une valeur de " pointage » (" pointing out »; Léard 1992; Bergen et Plauché 2001; De Cesare 2011), possiblement fondée sur une relation stéréotypique (Delahaie 2013). Son fonctionnement discursif repose sur l'articulation d'une dimension introductive et conclusive, assurant la cohésion textuelle (Auchlin 1981), dont la portée serait appréciative, marquant un pseudo-ajustement ou une validation (Druetta 1993).

Quand on procède à des observations sur corpus ( $\mathrm{Col}$ et alii 2015) on peut certes constater l'évantail de sens et de valeurs, mais cet évantail peut malgré tout se replier sur un double rôle pour voilà : délimiter une scène verbale (l'espace intersubjectif mis en place dans l'énonciation) et introduire des éléments sur la scène. D'après nous, ces deux rôles s'exercent à deux niveaux différents dans la mesure où l'un semble plus fondamental que l'autre même s'il n'est pas le plus fréquent dans les corpus observés : introduire des éléments sur la scène verbale, ce qui contribue fondamentalement à la délimitation de la scène en participant à sa structuration interne (Col et alii 2015).

Dans Col et alii (2015), nous avons proposé pour voilà et selon le principe de convocation-évocation l'instruction suivante :

- vollà : convoque sur la scène verbale des éléments dispersés (entités ou procès);

évoque leur regroupement dans un ensemble perceptible

Cette formulation tient compte du comportement de l'unité observé à travers les corpus analysés et repose sur l'hypothèse du regroupement d'informations (ou " grouping ") esquissée plus haut. Ce que nous appelons « éléments dispersés » dans la définition de l'instruction sémantique de voilà peut recouvrir différentes situations. Un extrait comme le suivant ${ }^{14}$ illustre par exemple le cas où voilà annonce le regroupement de l'ensemble d'informations qui est présenté dans le reste du discours (être recherché par la police, ne pas être l'auteur de l'assassinat, décidé à être interrogé par la police, ainsi que la relation concessive établie entre les deux dernières informations):

(7) Mais voilà, il est depuis recherché par la police dans le cadre d'une enquête sur une affaire de meurtre. Mais si John McAfee assure ne pas être l'auteur de cet assassinat, il n'est néanmoins pas décidé à se laisser interroger par la police.

Dans cet autre exemple, voilà vient plutôt réunir deux informations (téléphoner et emmener un costume) en réaction à ce que dit l'interlocuteur :

(8) L1 : et ben je vais téléphoner à Marguerite, je vais lui demander de t'emmener

un costume

L2 : en attendant euh

L1 : en attendant tu vas t'asseoir ici et je vais te chercher quelque chose, voilà.

Rappelons que l'instruction de voilà est basée sur un double mouvement de convocation-évocation, ainsi le sens que prend cette unité en contexte dépend aussi du sens qu'apportent des unités co-présentes dans l'énoncé. La formulation de l'instruction ne dit effectivement rien sur la distinction entre les différents sens de voilà. Suivant les contextes et les relations qu'entretient l'unité avec les autres unités linguistiques et leurs instructions, le regroupement des éléments peut ainsi prendre différentes formes. Il peut par exemple se faire en introduisant des éléments nouveaux sur la scène et en les intégrant dans cette représentation partagée. C'est ce que nous avons vu dans l'exemple (7); on a alors affaire en ce cas au rôle introductif de voilà. Le 
regroupement des éléments peut par ailleurs se faire en rassemblant simplement les éléments dispersés en vue de leur intégration dans la représentation partagée. On a alors affaire au rôle conclusif de voilà et ce cas correspond plutôt à l'exemple (8).

L'instruction de voilà étant maintenant définie, nous pouvons reprendre l'exemple (6) et proposer de le décomposer en unités linguistiques sur le modèle de l'analyse de l'exemple (1) faite plus haut :

voilà la photo que je P.C. (passé composé) préférer

Moment 1 : La première unité qui se présente à la perception est une unité dont le statut et la catégorie ne sont pas définissables dans un premier temps : volLÀ. On vient de faire l'hypothèse que cette unité, une fois perçue, convoque un ensemble d'éléments dispersés et évoque leur regroupement. Mais à ce stade de la perception de l'énoncé et de la construction de son sens, cette unité ne peut rien convoquer de tel. Sa catégorie n'est pas totalement définissable de toute façon, et même si l'apport sémantique de voILÀ va pouvoir se faire rapidement (comme pour la plupart des unités linguistiques), cette unité va être mise en attente et traitée complètement après les autres; elle sera donc réanalysée une fois les autres unités traitées.

L'unité LA va être également perçue et mise en attente, mais pour d'autres raisons. Comme nous l'avons montré dans Col et alii (2010), l'unité LA convoque une notion d'entité (notée « $\mathrm{e}$ » dans la figure ci-dessous) et évoque une occurrence de cette notion (notée « $\mathrm{E} »)$ obtenue par fléchage (noté « $\mathrm{fl} »)$. Le fléchage se définit comme une opération métalinguistique de détermination qui permet d'identifier une occurrence extraite d'un domaine notionnel (Culioli 1990). LA, dans la mesure où cette unité est catégorisable comme un article défini, évoque également que l'entité obtenue est issue d'une scène précédente, ou de la situation d'énonciation par une relation partie-tout. Mais à ce moment de la perception de l'énoncé et de la construction du son sens, LA est en attente d'une notion d'entité : aucune notion d'entité n'est effectivement présente sur la scène verbale et il faudra attendre le traitement d'une unité comme Рното pour que LA puisse jouer son rôle, ainsi que voILÀ.

Moment 2: Ce moment correspond à la perception de Рното et à son traitement. Cette unité linguistique joue un rôle lexical et comme toutes les unités lexicales, elle convoque une notion (que l'on peut désigner ici par /image/) et évoque une notion d'entité, c'est-à-dire une entité qui n'est pas complètement stabilisée au niveau sémantique (polysémie encore présente: "photo» peut prendre le sens d'«image imprimée sur papier » par exemple ou le sens de "vision d'une situation », etc) mais aussi au niveau de son statut d'entité "pleine». Sous l'effet de l'unité LA qui est présente sur la scène et qui attend une notion d'entité pour jouer son rôle dans la construction du sens et donner son instruction, cette notion d'entité évolue très vite vers une entité en grande partie stabilisée $e_{1}$ : dit autrement, LA convoque $e_{1}$ et évoque une entité $E_{1}$ obtenue par fléchage. Il est clair également que ce traitement rapide de Рното est aussi lié à son statut d'unité présente sur une scène précédente donc déjà en grande partie traitée et reconnaissable. On obtient ainsi une nouvelle entité désignée linguistiquement par LA РНото même si à ce stade cependant, LA n'est pas traitée complètement car cette unité indique qu'on a une entité obtenue par fléchage, or rien ne justifie complètement le fléchage à ce stade... («Quelle photo?»). Cette nouvelle entité entre alors dans la construction du sens de voILÀ comme on le voit ensuite. 
Moment 3 : Ce moment est un moment-clé dans la construction de la scène verbale car voILÀ peut apporter sa contribution et convoquer un élément, et simultanément l'entité $\mathrm{e}_{1}$ va se stabiliser encore davantage vers une entité pleine $\left(\mathrm{E}_{1}{ }^{15}\right)$, ce qui avait été amorcé au moment 2. C'est aussi un moment-clé pour une autre raison: l'interaction de ces trois premières unités permet l'émergence d'un sens qui repose sur la dimension déictique de vollà et que l'on pourrait paraphraser par «une entité "photo » est montrée dans la situation de locution», en conformité avec son instruction (rendre perceptibles des éléments dispersés).

La perception de QUE va pourtant modifier la scène assez profondément même si elle ne remet pas en cause radicalement le profil issu du moment précédent.

Moment 4 : l'unité QUE est perçue mais elle va être en fait immédiatement mise en attente car son instruction est d'introduire une nouvelle scène avec un changement de point de vue. QUE fait effectivement partie d'une famille d'unités dont l'instruction générale est de relier des paquets d'éléments plus ou moins grands entre différentes scènes. Sur la scène en construction est uniquement présent un complexe nominal rendu perceptible (grâce à voilà) et rien de plus. Si des liens existent avec d'autres scènes, et il en existe, ce sont essentiellement les liens créés autour de la présence de LA, comme on l'a vu, ainsi que ceux accompagnant vollà qui implique une relation de monstration et donc la présence même non marquée d'un interlocuteur. L'instruction donnée par QUE est de mettre en place une nouvelle scène intégrée dans une première scène et, suivant son statut syntaxique (en gros suivant l'opposition pronom relatif / conjonction de subordination), l'intégration de cette nouvelle scène est plus ou moins forte. Quand son traitement pourra s'effectuer, et donc quand QUE sera identifié comme un pronom relatif, l'intégration sera forte dans la mesure où cette unité va évoquer la mise en relation d'une entité avec une nouvelle scène sur laquelle cette entité est saillante. À ce moment-là est présente sur la scène une entité qui "demande " pour ainsi dire à être traitée car elle est perçue comme déterminée ("la photo ») mais rien ne justifie encore totalement ce degré de détermination ; seule l'action de voILÀ permet de traiter ce complexe nominal et donc de justifier le degré de détermination grammaticale. QUE peut convoquer cette entité $\mathrm{E}_{\text {photo }}$ qui est présente sur la scène initiale mais elle ne peut rien en faire car la nouvelle scène que QUE est censé relier à l'entité $\mathrm{E}_{\text {photo }}$ 'est pas encore construite. Les autres unités linguistiques devront être traitées avant QUE en tout cas afin que cette dernière puisse effectuer son travail de construction.

Moment 5 : Quand apparait à la perception l'unité J-, celle-ci va mettre en œuvre une partie de son instruction. En tant que pronom effectivement, le rôle de cette unité est de convoquer une entité existante et de la ré-évoquer sur la scène. Il est évident que pour le pronom JE (même sous sa forme J-), il s'agit de l'entité correspondant à l'actualisation d'une représentation du locuteur sur la scène. Mais ce travail n'est que partiel : en dehors de l'identification du locuteur et de son évocation (qui permet d'introduire une nouvelle entité sur la scène $E_{2}$ Pedro ainsi qu'un rôle agentif), elle ne peut être mise en relation avec un procès car il n'y en a pas sur la scène à ce moment-là (PRÉFÉRER n'a pas en core été perçu ni traité).

78 Moment 6: Les deux dernières unités, le passé composé (PC) et PRÉFÉRER, vont être traitées très rapidement et ceci pour plusieurs raisons. PRÉFÉRER est immédiatement précédée par le verbe «avoir» sous la forme AI. La séquence AI PRÉFÉRÉ sera traitée 
globalement dans notre modèle, comme la forme conjuguée au passé composé du verbe PRÉFÉRER. Le passé composé est un temps du passé qui se construit relativement à l'intervalle d'énonciation à partir de deux marqueurs : le participe passé qui désigne le procès lui-même et l'auxiliaire qui exprime l'état résultant de ce procès. À ces deux formes, nous faisons correspondre deux intervalles de référence, l'intervalle de référence étant dans le modèle de Gosselin (1996) la fenêtre de monstration à partir de laquelle l'énonciateur donne à voir le procès sur la scène verbale. Le premier intervalle de référence [I II] coïncide avec les bornes [B1 $\mathrm{B} 2]$ du procès lié à " préférer » et le deuxième intervalle de référence [I'II'], associé à l'auxiliaire « avoir » [B'1 B'2] coïncide dans la mesure où « avoir » est au présent, avec l'intervalle d'énonciation [01 02]. Nous reprenons ici le formalisme et la notation de Gosselin (1996) :

$01 \quad 02$

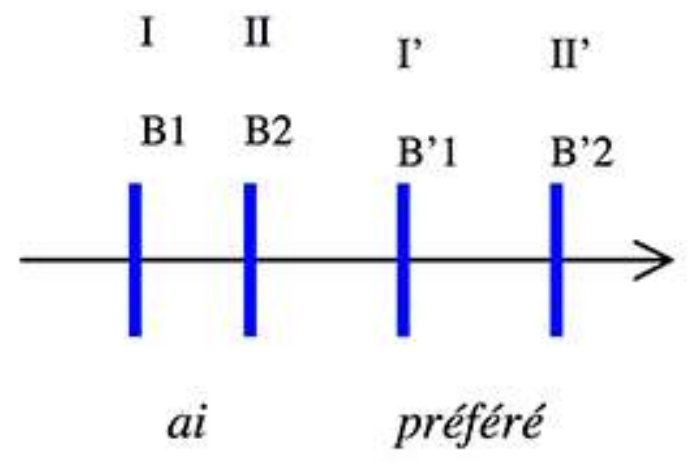

Fig. n 2 : sémantique du passé composé (adapté de Gosselin 1996)

Par ailleurs, PRÉFÉRER sera traité avant PC car l'instruction fournie par cette unité (unité lexicale) est de convoquer une notion («préférence d'un élément à un autre ») immédiatement perçue sous la forme d'une notion de procès $p$, et d'évoquer cette notion sur la scène. Une fois évoquée, cette notion de procès va être profilée vers un procès mais ce profilage est lié à la convocation effective d'un agent et celle, potentielle, d'un objet car elle a besoin d'instancier son schéma actanciel. À cette étape, une seule entité $\left(\mathrm{E}_{\text {Pedro }}\right)$ peut être convoquée car elle est présente sur la scène (et même sur les deux scènes) et elle est maintenue en attente de traitement. Elle va donc pouvoir être convoquée et accrochée à la notion de procès. Mais si la notion de /préférence/ gagne ici en détermination processuelle, elle ne peut pas être complètement traitée car elle ne bénéficie pas de détermination aspecto-temporelle à cette étape du traitement. C'est effectivement la perception de l'unité PC (passé composé) qui va permettre d'avancer dans la processualisation ${ }^{16}$ de $p$. Le passé composé convoque un procès $p$ et évoque deux "intervalles de monstration » c'est-à-dire deux fenêtres temporelles à travers lesquelles sont présentés les éléments de la scène verbale. Le premier intervalle est centré sur l'instant de l'énonciation, et le second est un intervalle antérieur englobant le procès $p$ dans sa totalité. Cette double évocation permet de rendre compte des deux sens du passé composé, à la fois le sens d'accompli ( Il a fini son sandwich depuis une heure ») et le sens d'aoriste ("Il a fini son sandwich il y a une heure ») (Gosselin 2005). Pour être complet, le traitement du complexe verbal a besoin de convoquer une dernière unité qui lui permet d'instancier entièrement son schéma actanciel et de se transformer en procès " plein » (noté $\mathrm{P}$ ). Il se trouve qu'une telle entité est présente sur 
la scène car elle a été évoquée par QUE : il s'agit de $E_{\text {photo }}$. La notion de procès $p$ va ainsi convoquer cette entité et évoluer vers un procès $\mathrm{P}$. À ce stade de la construction de la scène, QUE peut ainsi être totalement traitée, grâce à une double action des unités : AI PRÉFÉRÉ recherche un élément pour finaliser sa relation actancielle et simultanément l'unité QUE, en attente de traitement, a besoin d'un procès pour effectuer tout son travail de mise en relation de deux scènes car elle évoque une entité qui peut finaliser la relation actancielle en suspens. La scène correspondant à la subordonnée relative est mise en place dans la scène initiale qui correspond à la proposition principale ; c'est à ce moment que voILÀ va être réanalysée.

Moment 7: L'unité voILÀ, qui a déjà été traitée et qui a effectué son rôle dans la construction de la scène initiale, va convoquer les nouveaux éléments qui se trouvent présents sur la scène imbriquée. Ces éléments (un procès $P_{1}$, une entité $E_{2 \text { Pedro }}$ et leur mise en relation grâce à l'instruction aspecto-temporelle) constituent un ensemble à regrouper et à associer à l'entité $\mathrm{E}_{\text {photo }}$; ainsi, conformément à son instruction, voILÀ convoque $\mathrm{E}_{\text {photo }}$ et la scène 2 sur laquelle $\mathrm{E}_{\text {photo }}$ est également présente (mais sous forme d'évocation par QUE) et elle évoque le regroupement de la scène 2 avec l'entité $\mathrm{E}_{\text {photo }}$ pour les rendre perceptibles (noté «Group. E1 - scène 2 " dans la Figure $\mathrm{n}^{\circ} 3$ cidessous).

Comme pour l'exemple (1), nous proposons une schématisation qui reprend les différentes étapes du traitement de l'énoncé suivant l'ordre de perception des unités linguistiques présentes. Ce schéma illustre et synthétise ces étapes et cherche à montrer le cheminement de la construction du sens de l'énoncé ${ }^{17}$ :

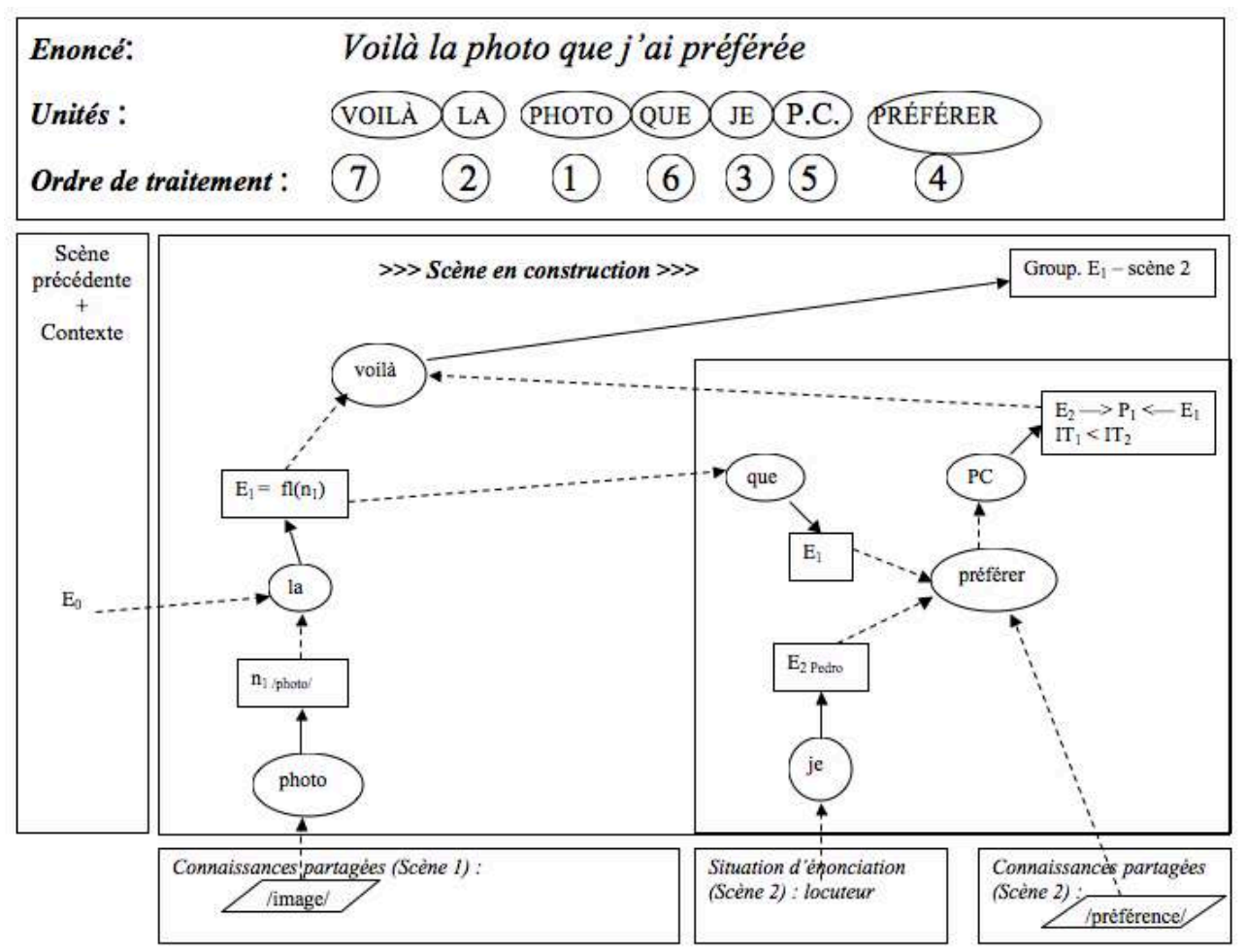

Fig. $n^{\circ} 3$ : progression sémantique de l'énoncé : « Voilà la photo que j'ai préfèrée » 


\section{En guise de conclusion : questions en suspens et points à débattre}

Notre contribution tente de mettre sur pied une vision à la fois différente de la notion d'instruction et de point de vue développée par Pierre-Yves Raccah tout en adhérant à la philosophie générale que ce dernier a devéloppée dans ses travaux. Pour autant, la notion d'instruction doit d'après nous être généralisée à toutes les unités linguistiques au-delà des "articulateurs ", même si ces derniers jouent un rôle incontestablement central.

$\mathrm{Au}$ vu de cette présentation, plusieurs grandes questions restent néanmoins - et tout naturellement - en suspens. La première consiste dans la mise en place de ce formalisme, assez lourd, notamment lorsqu'il s'agira d'étendre ces travaux au-delà du seul cadre de la proposition ou de la subordination simple comme dans l'exemple français présenté plus haut. La question de l'ordre de traitement est par ailleurs une question essentielle dans la mesure où elle repose sur ce mode de calcul compositionnel gestaltiste qui fait l'originalité de notre approche. La question de la porosité des classes grammaticales est également une question centrale, notamment vue sous un angle continuiste. Même si une réponse partielle a été apportée à travers l'étude d'une unité comme « over » dans Col et Poibeau (2014), on peut par exemple se poser la question de savoir comment chercher à rendre compte de la très faible distinction entre des unités comme les prépositions et les particules. La dernière question concerne la prise en charge de l'oral. Il s'agit de savoir quel est le rôle de la prosodie et de l'intonation dans l'interaction avec les instructions des unités linguistiques. Cette problématique a déjà été abordée dans d'autres travaux ( $\mathrm{Col} 2009,2010 \mathrm{a}$ ), mais le traitement systématique des données prosodiques dans la définition des instructions sémantiques est encore en projet.

\section{BIBLIOGRAPHIE}

Auchlin Antoine, «Mais heu, pis bon, ben alors voilà, quoi! Marqueurs de structuration de la conversation et complétude ", Cahiers de linguistique française $\mathrm{n}^{\circ}$ 2, 1981, pp. 141-160.

Benveniste Émile, Problèmes de linguistique générale, vol. 1, Paris, Gallimard, 1966.

Bergen Benjamin et Plauché Madelaine, «Voilà, voilà : Extensions of Deictic constructions in French », in Cienki Alain, Luka Barbara et Smith Michael (éds), Conceptual and Discourse Factors in Linguistic Structure, CSLI, 2001, pp. 45-61.

Brunot Ferdinand et Bruneau Charles, Précis de grammaire historique de la langue française, Paris, Masson et cie, 1969.

Col Gilles, «Rôles de until et de by dans la mise en scène verbale », in Paillard Michel (dir.), Préfixation, préposition, postposition. Étude de cas, Rennes, Presses Universitaires de Rennes, 2008a, pp. 145-161. 
Col Gilles, « Modèle instructionnel du rôle des unités linguistiques dans la construction dynamique du sens ", in Chuquet Jean (dir.), Le Langage et ses niveaux d'analyse. Cognition, production de formes, production du sens, Rennes, Presses Universitaires de Rennes, 2008b, pp. 45-60.

Col Gilles, « Activation et saillance d'unités grammaticales. Quelle place pour l'intonation dans la construction dynamique du sens ? », Travaux linguistiques du CerLiCO n² 22, 2009, pp. 59-79.

Col Gilles, « Remarques sur le rôle de l'intonation dans le traitement de l'information », Corela, HS 9 : « Parole », 2010a. Accessible en ligne : http://corela.revues.org/1016.

Col Gilles, « Correspondance et mixage d'espaces mentaux dans la construction dynamique du sens », Mémoires de la Société de Linguistique de Paris vol. 18, Louvain, Peeters, 2010b, pp. 53-73.

Col Gilles (soumis), Un Modèle instructionnel pour la sémantique, 2016.

Col Gilles et Poibeau Thierry, « An Instruction-Based Analysis of Over », Language and Cognition vol. $6, \mathrm{n}^{\circ} 3,2014$, pp. 370-407.

Col Gilles, Aptekman Jeanne, Girault Stéphanie et Victorri Bernard, « Compositionnalité gestaltiste et construction du sens par instructions dynamiques », CogniTextes, vol. 5, 2010. Accessible en ligne : http://cognitextes.revues.org.

Col Gilles, Aptekman Jeanne, Girault Stéphanie et Poibeau Thierry, « Gestalt Compositionality and Instruction-based Meaning Construction », Cognitive Processing, vol. 13, $\mathrm{n}^{\circ}$ 2, 2012, pp. 151-170.

Col Gilles, Danino Charlotte et Rault Julien, « Éléments de cartographie des emplois de voilà en vue d'une analyse instructionnelle ", Revue de Sémantique et de Pragmatique, n 37, 2015, pp. 37-59.

Col Gilles, Danino Charlotte, Rault Julien et Knutsen Dominique (à par., 2016), « Rôle de voilà dans l'affirmation : valeur confirmative et marque d'intégration d'informations », in Testi e Linguaggi, Università di Salerno, Italia.

Culioli Antoine, Pour une linguistique de l'énonciation, vol. 1. Ophrys, 1990.

Culioli Antoine, Pour une linguistique de l'énonciation, vol. 3. Ophrys, 1999.

De Cesare Anna-Maria, «L'italien ecco et les français voici / voilà. Regards croisés sur leurs emplois dans les textes écrits ", Langages $n^{\circ}$ 184, 2011, pp. 51-67.

Delahaie Juliette, « Vers une analyse sémantique (presque) unitaire des multiples emplois de voilà à l'écrit et à l'oral », Revue de Sémantique et de Pragmatique nos 33-34, 2013, pp. 99-120.

Druetta Ruggero, Étude de voilà non-constructeur, Testi di Laurea, Universita del Torino, manuscrit dactylographié, 1993.

Fauconnier Gilles, « Subdivision cognitive », Communications nº 53, 1991, pp. 229-247.

Fauconnier Gilles, Mappings in Thought and Language, Cambridge University Press, 1997.

Gilbert Eric, « Vers une analyse unitaire des modalités », Cahiers de Recherche en Grammaire

Anglaise $\mathrm{n}^{\circ}$ 8, 2001, pp. 23-99.

Gosselin Laurent, Sémantique de la temporalité en français. Un modèle calculatoire et cognitif du temps et de l'aspect, Duculot, 1996.

Gosselin Laurent, Temporalité et modalité, Duculot, 2005.

Gosselin Laurent, Les modalités en français, Rodopi, 2010.

Grenoble Lenore, A. et Riley Matthew, "The rôle of deictics in discourse coherence: French voici / voilà and Russian vot / von ", Journal of Pragmatics n² 25, 1996, pp. 819-838. 
Grevisse Maurice, Le Bon usage, Louvain, Duculot, 1980.

Guillaume Paul, La Psychologie de la forme, Paris, Flammarion, [1919] 1979.

Hansen Maj-Britt Mosegaard, « Alors and donc in spoken French: a reanalysis », Journal of Pragmatics nº 28, 1997, pp. 153-187.

Huddleston Rodney et Pullum Geoffrey K., The Cambridge Grammar of the English Language, Cambridge, Cambridge University Press, 2002.

Kamp Hans et Reyle Uwe, From Discourse to Logic. An Introduction to the Modeltheoretic Semantics of Natural Language, Formal Logic and Discourse Representation Theory, Kluwer, Dordrecht, 1990.

Langacker Roland, Foundation of Cognitive Grammar, vol. 1. Stanford, Cal. Stanford University Press, 1987.

Le Bidois Georges, Syntaxe du français moderne. Ses fondements historiques et psychologiques, Paris, Éditions Auguste Picard, 1956.

Le Ny Jean-François, « Éléments de psycholinguistique cognitive : des représentations à la cognition », in Fuchs Catherine (dir.), La Linguistique cognitive, Paris, Ophrys, 2004, pp. 155-170.

Le Ny Jean-François, Comment l'esprit produit du sens, Paris, Odile Jacob, 2005.

Léard Jean-Marcel, Les Gallicismes, Louvain, Duculot, 1992.

Moignet Gérard, « Le verbe voic-voilà », Travaux de linguistique et de littérature $n^{\circ} 8,1969$, pp. 189-202.

Morin Yves-Charles, " On the two french subectless verbs 'voici' and 'voilà' " , Language vol. 61, $\mathrm{n}^{\mathrm{0}}$ 4, 1985, pp. 777-820.

Narjoux Cécile, "C'est cela que c'est la tragédie, ou les présentatifs dans Électre de Giraudoux », Information grammaticale $\mathrm{n}^{\circ} 96,2003$, pp. 43-53.

Paillard Denis, «À propos des verbes « polysémiques » : identité sémantique et principes de variation », Syntaxe et Sémantique $\mathrm{n}^{\circ} 2,2001$, pp. 99-120.

Pariollaud Fanelly, Verbes. Questions de sémantique, Rennes, Presses Universitaires de Rennes, 2008.

Porhiel Sylvie, « The presentative voici/voilà - Towards a pragmatic definition », Journal of Pragmatics $\mathrm{n}^{\circ} 44,2012$, pp. 435-452.

Quirk Randolph, Greenbaum Sidney, Leech Geoffrey et Svartvik Jan, A Comprehensive Grammar of the English Language, London, Longman, [1985] 1994.

Raccah Pierre-Yves, « Une description de l'excessivité en sémantique des points de vue », Travaux Linguistiques du CerLiCO 18, n 18, 2005, pp. 171-190.

Raccah Pierre-Yves, « La sémantique des points de vue, un outil utile ? Conférence à l'Université d'Orléans, 24 janvier 2011.

Talmy Len, Toward a Cognitive Semantics, MIT Press, 2000.

Victorri Bernard, La Polysémie, Paris, Hermès, 1996.

Victorri Bernard, « Le sens grammatical », Langages n 136, 1999, pp. 85-105.

Wagner Robert-Léon et Pinchon Jacqueline, Grammaire du français classique et moderne, Paris, Hachette, 1962. 


\section{NOTES}

1. «Une phrase constitue un tout, qui ne se réduit pas à la somme de ses parties; le sens inhérent à ce tout est réparti sur l'ensemble des constituants. Le mot est un constituant de la phrase, il en effectue la signification; mais il n'apparait pas forcément dans la phrase avec le sens qu'il a comme unité autonome ». (Benveniste 1966 : 123-124).

2. Voir Discourse Representation Theory de Kamp et Reyle (1990).

3. Une grande partie des recherches développées dans ces lignes est issue de travaux portant sur l'anglais (Col 2008a, 2008b, 2009, 2010a, 2016, Col et Poibeau 2014). Des analyses portant sur le français sont présentées dans Col, Aptekman, Girault et Victorri (2010) ; Col, Aptekman, Girault et Poibeau (2012).

4. Suivant les auteurs, cette distinction est plus ou moins maintenue (Talmy 2000) ou mise en perspective continuiste (Victorri 1999).

5. Ceci est loin de s'opposer à la reconnaissance d'un sens lexical et d'un sens grammatical. Comme nous l'avons mentionné, l'opposition entre sens lexical et sens grammatical ne saurait être comprise comme opérant une coupure nette entre deux types d'unités, puisqu'une même unité peut tout à fait avoir à la fois un sens lexical et un sens grammatical. Cependant, nous allons plus loin ici, en considérant que les instructions ne sont pas l'apanage du seul sens grammatical, comme nous allons nous appliquer à le montrer.

6. Victorri $(1996,1999)$; Col (2008a, 2008b, 2009, 2010a, 2016); Col et alii $(2010,2012)$.

7. La distinction entre « entité » et «procès » est proche de celle proposée par Langacker (1987) entre « chose » («thing ) ) et procès (« process»).

8. Sur cette question, l'approche développée par Gosselin (2005) est proche de la nôtre, notamment à travers la notion de simulation de perception.

9. «Parler de notion, c'est parler d'un ensemble que l'on peut exprimer, par exemple, par « lire ; lecture ; livre ; lecteur ; bibliothèque; etc. » et c'est dire qu'on ne peut pas ramener les choses à une unité lexicale ; celle-ci va servir de porte-manteau, d'entrée, mais c'est tout. [...] Le problème est que la notion est quelque chose de virtuel et de productif. Elle n'est pas donnée dans toutes ses acceptions et c'est pour cela qu'elle ne peut pas correspondre à une unité lexicale. Elle est un générateur d'unités lexicales; elle définit une classe d'opérations reliées. Elle désigne ». (Culioli 1990 : 53-54).

10. La lettre capitale indique que l'on a affaire à une entité et plus à une "notion d'entité », et la lettre « $\mathrm{n}$ » en indice indique que cette entité est issue d'une notion.

11. Rappelons qu'une grande partie des recherches développées dans ces lignes est issue de travaux portant sur l'anglais (Col 208a, 2008b, 2009, 2010a, 2016 ; Col et Poibeau 2014 ; voir note 3).

12. Comme nous l'avons précisé, il s'agit ici de présenter des travaux en cours plutôt qu'achevés, c'est pourquoi ces formes schématiques, ainsi que la grammaire instructionnelle à laquelle elles doivent donner lieu, doivent être appréhendés comme en construction. De la même façon, l'analyse de l'exemple présentée ci-après se situe dans la continuité d'analyses d'énoncés contenant ALWAYS (Col 2009, 2010a), afin de mettre en évidence à la fois la validité de la forme schématique de cette unité, la validité de l'hypothèse défendue ici ainsi que de sa méthodologie.

13. Dans la littérature, -EN est la notation du participe passé anglais.

14. Voir Col et alii (à par. 2016).

15. La notation développée utilisée est la suivante $: \mathrm{E}_{1}: \mathrm{fl}\left(\mathrm{e}_{1}\right)$ avec « $\mathrm{fl}$ » pour « fléchage ».

16. Par " processualisation », nous entendons dans notre modèle : "tranformation en procès ».

17. Comme pour la Figure $n^{\circ} 1$, les flèches en pointillés signalent ce que les unités convoquent sur la scène et les flèches pleines signalent ce qu'elles évoquent. 


\section{RÉSUMÉS}

L'hommage aux travaux de Pierre-Yves Raccah que nous proposons dans ce texte prend la forme d'une série de propositions cherchant à développer une sémantique instructionnelle pour décrire la construction dynamique et progressive du sens d'un énoncé. Une définition de la notion d'instruction est présentée et comparée avec d'autres définitions de cette notion. Une formulation de cette notion en termes de convocation-évocation est ensuite introduite. Elle s'accompagne d'une série de principes de traitement de ces instructions dans un énoncé. Pour finir, nous exposons deux exemples de traitement complet des instructions d'un énoncé, en anglais et en français, afin d'illustrer comment on peut tenter de décrire la construction du sens par instructions dynamiques.

The tribute to Pierre-Yves Raccah's works we would like to offer in our article is a series of propositions seaking to develop an instructional model to describe the gradual, dynamic construction of the meaning of an utterance. A definition of the notion of instruction is first presented and compared with other definitions. A formulation of this notion in terms of convocation-evocation is then introduced, along with a series of principles ruling the processing of these instructions in an utterance. Finally, we display the complete processing of two examples, in English and in French, so as to illustrate how we can describe the construction of meaning by dynamic instructions.

\section{INDEX}

Mots-clés : Instruction sémantique, convocation-évocation, ordre de traitement, perception du sens

Keywords : Semantic instruction, convocation-evocation, processing order, perception of meaning

\section{AUTEURS}

\section{GILLES COL}

Laboratoire LATTICE

PSL Research University

CNRS \& ENS \& U. Sorbonne nouvelle

\section{JEANNE APTEKMAN}

Laboratoire LATTICE

PSL Research University

CNRS \& ENS \& U. Sorbonne nouvelle 